\title{
Questes
}

Revue pluridisciplinaire d'études médiévales

\section{Introduction. La prophétie médiévale : théologie et fiction, politique et langage}

\section{Servane Rayne-Michel}

\section{(2) OpenEdition}

1 Journals

Édition électronique

URL : http://journals.openedition.org/questes/3387

DOI : 10.4000/questes.3387

ISSN : 2109-9472

Éditeur

Les Amis de Questes

\section{Édition imprimée}

Date de publication : 15 juin 2014

Pagination : $3-28$

ISSN : 2102-7188

\section{Référence électronique}

Servane Rayne-Michel, « Introduction. La prophétie médiévale : théologie et fiction, politique et langage ", Questes [En ligne], 28 | 2014, mis en ligne le 15 juin 2014, consulté le 25 septembre 2020. URL: http://journals.openedition.org/questes/3387 ; DOI : https://doi.org/10.4000/questes.3387 


\title{
Introduction
}

\section{La prophétie médiévale : théologie et fiction, politique et langage}

\author{
Servane RAYNE-MICHEL \\ Université Paris-Sorbonne (Paris IV)
}

Une synthèse des richesses et contradictions de la prophétie médiévale apparaît dans la figure de Merlin - bien au-delà du simple enchanteur auquel a pu le réduire la tradition cinématographique et littéraire contemporaine ${ }^{1}$. Prophète des rois de Bretagne et prophète du Graal, inscrit dans l'espace politique et historiographique par les chroniques du XII ${ }^{\mathrm{e}}$ siècle ${ }^{2}$ autant que dans l'espace littéraire des romans arthuriens $^{3}$, il illustre une tension qui parcourt tout l'Occident $\mathrm{du} \mathrm{VI}^{\mathrm{e}}$ au $\mathrm{XIV}^{\mathrm{e}}$ siècles : à travers son personnage, les romans comme les chroniques problématisent l'histoire. Malgré leur caractère hybride - politiques à l'origine, elles mordent sur le territoire du religieux, et ce même dans la fiction-, ses prophéties ont circulé sans réserve de la part des autorités ecclésiastiques pendant tout le Moyen Âge; ce n'est qu'au concile de Trente qu'elles furent mises à l'index des ouvrages condamnés. Cette

${ }^{1}$ Cf. L'Enchanteur de René Barjavel (Paris, Denoël, 1984) ou le Merlin de Walt Disney (1963), pour ne citer que les plus connus.

${ }^{2}$ Le prophète Merlin doit le début de sa carrière littéraire à Geoffroy de Monmouth, chroniqueur anglo-normand qui lui consacre un ouvrage, la Vita Merlini (Vie de Merlin par Geoffrey de Monmouth, éd. Christine Bord et Jean-Charles Berthet, dans Le devin maudit. Merlin, Lailoken, Suibhne. Textes et études, éd. Philippe Walter, Grenoble, ELLUG, coll. « Moyen Âge européen », 1999, p. 49-171).

${ }^{3}$ C'est Robert de Boron qui lui donne un statut romanesque dans son Merlin (Robert de Boron, Merlin, roman $d u$ XIII siècle, éd. Alexandre Micha, Genève, Droz coll. « Textes littéraires français », 281, 1979). 
ambivalence, qui caractérise la prophétie au Moyen Âge, remonte comme le souligne l'historien André Vauchez aux origines du christianisme : alors que l'Incarnation du Fils de Dieu accomplit de manière totale et définitive les prophéties de l'Ancienne Alliance, et rend caduque la fonction prophétique telle qu'elle existait dans le peuple élu, le Nouveau Testament clôture la Révélation divine, tout en reconnaissant l'existence du don de prophétie parmi les « charismes ${ }^{4} »$ accordés par le Saint-Esprit aux fidèles à partir de la Pentecôte. La prophétie, dès lors, est moins prédiction d'événements futurs qu'interprétation des «signes des temps», compréhension des Écritures et de leur signification pour le chrétien dans son temps, actualisation de l'unique Révélation pour le bien spirituel des fidèles. La prophétie peut donc être revendiquée par tout chrétien comme une inspiration divine - d'où la difficulté pour les autorités ecclésiastiques et politiques de contrôler les interventions supposées de Dieu dans son Église, lorsqu'elles sont revendiquée par des fidèles sans autorité hiérarchique $^{5}-$; d'où également le potentiel littéraire de cet esprit prophétique, au fondement d'une culture qui repose sur l'autorité de la Parole : celui qui écrit la prophétie est reçu comme traduisant et mettant à la portée de tous une inspiration dont il est le bénéficiaire singulier. Que la prophétie se présente comme la transcription fidèle d'un langage divin ou comme la médiation nécessaire pour transmettre aux hommes une révélation inarticulée, le langage est chargé d'une puissance de

${ }^{4} C f$. I Corinthiens XII, 8-10. À côté du don de prophétie se trouvent les dons de discernement et d'interprétation des Écritures.

${ }^{5}$ André Vauchez note à cet égard un tournant dans l'histoire de la prophétie médiévale, lorsque le pape Eugène III et saint Bernard reconnaissent officiellement les visions de l'abbesse Hildegarde de Bingen (en 1147). Désormais, la fonction prophétique, jusque là réservée en exclusivité aux membres de la hiérarchie ecclésiastique (depuis la crise montaniste, au $\mathrm{IV}^{\mathrm{e}}$ siècle), est reconnue comme légitime parmi les membres les plus humbles du peuple de Dieu ( $c f$. André Vauchez, Saints, prophètes, visionnaires: le pouvoir surnaturel au Moyen Âge, Paris, Albin Michel, 1999, p. 115). 
signification censée dépasser les limites de l'imagination humaine ${ }^{6}$.

\section{Merlin et la prophétie politique}

À travers le personnage de Merlin apparaissent les principales dimensions de la prophétie politique au Moyen Âge.

\section{Prophétie politique et eschatologie}

Les prophéties attribuées au personnage de Merlin sont perçues avant tout comme l'annonce d'événements à venir dans l'histoire des puissances de ce monde - prédiction de règnes, d'ascensions et de chutes, d'alliances et de guerres. Leur origine en effet est politique, puisqu'elles apparaissent d'abord dans l'Histoire des rois de Bretagne, chronique écrite au début du $\mathrm{XII}^{\mathrm{e}}$ siècle par 1'anglo-normand Geoffroy de Monmouth ${ }^{7}$. Par la suite souvent traduites, réécrites ou réinventées, elles suscitent tout au long du Moyen Âge l'intérêt ou l'inquiétude de tous les pouvoirs en place, qu'ils soient politiques ou ecclésiastiques. Les Plantagenêt en particulier les promeuvent, y cherchant une légitimation de leur pouvoir ${ }^{8}$.

L'origine de la prophétie politique remonte d'un côté à l'Ancien Testament, avec l'exemple illustre du festin de Balthazar, au livre de Daniel - l'apparition, au cours d'un banquet donné par le roi de Babylone, d'une main venant écrire sur le mur trois mots inconnus, est interprétée par

\footnotetext{
${ }^{6}$ Il en va de même pour les auteurs de la Bible, dont les talents littéraires propres sont mis au service de la Révélation divine, unique à travers ses différentes formes.

${ }^{7}$ The "Historia regum Britanniae » of Geoffrey of Monmouth, éd. Neil Wright, Cambridge, Brewer, 1988.

${ }^{8}$ Sur l'exploitation du mythe arthurien par les Plantagenêt, voir Amaury Chauou, L'Idéologie Plantagenêt, Royauté arthurienne et monarchie politique dans l'espace Plantagenêt (XII ${ }^{e}-X I I I^{e}$ siècles), Rennes, Presses Universitaires de Rennes, 2001, en particulier p. 265-269; pour une étude plus vaste de la dimension politique des prophéties merliniennes voir Catherine Daniel, Les Prophéties de Merlin et la culture politique (XII $-X V I^{e}$ siècles), Turnhout, Brepols, 2006.
} 
le jeune israëlite comme une annonce de la chute du roi Balthazar ${ }^{9}$. Mais Daniel est également resté dans la mémoire médiévale comme le prophète biblique recevant par des songes des révélations divines sur la destinée de l'empire babylonien ${ }^{10}$. Les songes prophétiques trouvent dans l'Ancien Testament un autre modèle en la personne de Joseph, déporté israëlite en Égypte qui gagne la faveur de Pharaon en élucidant ses songes ${ }^{11}$. Le motif du songe royal expliqué par un personnage d'origine modeste ou obscure est récurrent dans le schéma de la prophétie politique - il se retrouve dans le Lancelot en prose et, à travers le prophète Merlin ou d'humbles ermites, dans tout le cycle arthurien où il reprend le plus souvent le caractère sombre de la prophétie de Daniel à Balthazar: le songe royal est généralement porteur d'une prophétie de mort et de destruction ${ }^{12}$.

C'est surtout l'Apocalypse de saint Jean qui donne à la prophétie la tonalité catastrophiste qui l'accompagnera durant tout le Moyen Âge; les signes cosmiques, politiques et moraux qui - selon une lecture littéraleaccompagneront la fin des temps, sont interprétés dans un sens politique par une tradition vivace tout au long du Moyen Âge. La chronologie des sept millénaires indiquée par Jean est sujette aux conjectures les plus diverses sur l'identité des dynasties qui ont pu ou pourront l'illustrer. Le

${ }^{9} C f$. Daniel v-VII.

${ }^{10} C f$. Daniel vII $s q$. Sur la postérité médiévale des songes de Daniel, voir en particulier Curt Ferdinand Bühler, "Two Middle English Texts of the Somnia Danielis », Early Books and Manuscripts, New York, Grolier Club, 1973, p. 555-563 ; éd. Lawrence T. Martin, Somniale Danielis. An edition of a Medieval Latin dream interpretation handbook, Frankfurt am Mein, Peter Lang, coll. " Lateinische Sprache und Literatur des Mittelalters », 10, 1981; Thierry Revol, «Incarnation et représentations : le prophète Daniel dans les drames liturgiques du XII ${ }^{\mathrm{e}}$ siècle », Entre l'ange et la bête. L'homme et ses limites au Moyen Âge, dir. Marie-Étiennette Bély, Jean-René Valette et Jean-Claude Vallecalle, Lyon, Presses universitaires de Lyon, 2003, p. 53-68.

${ }^{11} C f$. Genèse XLI, 1-36.

${ }^{12} C f$. François Berriot, "Rêver la mort, rêver la résurrection. Notes sur les clés des songes du Moyen Âge et de la Renaissance », Réforme, Humanisme, Renaissance, XIX, 37, 1993, p. 51-63; sur l'utilisation des rêves de mort dans la littérature arthurienne, voir Jehanne Joly, «Rêves prémonitoires et fin du monde arthurien », Fin des temps et temps de la fin dans l'univers médiéval, Senefiance, 33, Aix-enProvence, CUERMA, 1993, p. 261-284. 
drame de la fin des temps est en effet chez saint Jean rythmé par l'ouverture de sept sceaux, chacun représentant une période de mille ans. La septième s'ouvre avec la lutte entre Satan et le Christ, à l'issue de laquelle celui-ci, victorieux, enchaîne son adversaire dans l'abîme pour une période de mille ans. Cette période doit voir le règne du Christ sur terre tandis que les élus, ressuscités, connaîtront un Sabbat - temps de repos : le « septième jour»- avant le combat final, la résurrection de tous les hommes et le Jugement dernier. Ce millénaire de règne du Christ a fait l'objet de deux interprétations divergentes, qui sont fondamentales pour comprendre les évolutions de la prophétie au Moyen Âge ${ }^{13}$, son rôle politique aussi bien que les textes multiformes qu'elle a engendrés. La première interprétation, dite millénariste, voit dans le millénaire de règne terrestre du Christ l'annonce d'un temps de renouveau cosmique accompagné de paix politique et de prospérité pour l'Église ${ }^{14}$. La tradition prophétique qui adopte cette perspective millénariste témoigne de ce «vieux désir de faire éclater le cadre de la Révélation telle qu'elle est étroitement définie par l'Église ${ }^{15} »$; les élans prophétiques périodiquement renouvelés au cours du Moyen Âge témoignent paradoxalement aussi bien de cette insatisfaction face à la Révélation contenue dans les Écritures, que de la foi dans l'attention actualisée que Dieu porte aux hommes par l'intermédiaire de ses prophètes. L'espoir d'un retour à des temps paradisiaques se traduit dans le grand nombre de textes prophétiques qui, aux $\mathrm{XII}^{\mathrm{e}}-\mathrm{XIII}^{\mathrm{e}}$ siècles, prétendent identifier le souverain pacificateur des

\footnotetext{
${ }^{13}$ Le millénarisme constitue, selon André Vauchez, l'une des «trois formes principales du prophétisme », avec l'eschatologie et l'utopie (André Vauchez, Prophètes et prophétisme, Paris, Seuil, 2012, Introduction, p. 18).

${ }^{14}$ Sont à cet égard convoquées les prophéties d'Ézéchiel et d'Isaïe.

${ }^{15}$ Claude Carozzi et Huguette Taviani-Carozzi, La fin des temps. Terreurs et prophéties au Moyen Âge, Paris, Flammarion coll. « Champs », 446, 1999 [Paris, Stock, 1982], p. 12.
} 
temps derniers. Mais c'est surtout la figure de l'Antéchrist ${ }^{16}$ qui fait l'objet de vaticinations : sa venue, accompagnée de catastrophes cosmiques et de violences extraordinaires, fait l'objet d'attentes qui sont revivifiées à chaque période de crise. Les auteurs ou interprètes de prophéties cherchaient dans les événements contemporains - dans l'invasion arabe, notamment ${ }^{17}$ - des signes annonçant l'approche du septième millénaire, et dans les souverains hostiles à la papauté des figures de l'Antéchrist. Des oracles attribuées aux Sibylles antiques ${ }^{18}$ sont retrouvés ou même réinventés pour nourrir l'attente de la fin et identifier les souverains des derniers temps. Malgré les condamnations de l'Église, le millénarisme connaît un renouveau à partir du XIV ${ }^{\mathrm{e}}$ siècle, avec l'héritage de Joachim de Flore qui restera vivace jusqu'au $\mathrm{XVI}^{\mathrm{e}}$ siècle. Joachim revient à une interprétation historique de l'Apocalypse, dont il fait la clé d'une lecture théologique de l'histoire ${ }^{19}$. Ses prophéties, reprises, développées, réinterprétées, ont connu un succès immense : 1'autorité de Joachim de

${ }^{16}$ L'Antéchrist est celui qui doit venir avant le Christ ; les médiévaux, d'après la forme latine Antichristus, y identifiaient plus souvent dans ce nom le préfixe anti-, mettant ainsi l'accent sur l'opposition au Christ que symbolise l'Antéchrist (d'où le choix de Claude Carozzi et Huguette Taviani-Carozzide traduire ce terme, contrairement à l'usage, par celui d' « Antichrist », dans La fin des temps, op. cit.).

${ }^{17}$ Voir en particulier La Description des derniers temps du Pseudo-Méthode (Sermo de regnum cantium et in novissimis temporibus certa demonstratio, dans Sibyllinische Texte und Forschungen, éd. Ernst Sackur, augmentée par Raoul Manselli, Torino, Bottega d'Erasmo, 1976 [La Halle, Max Niemeyer, 1898]). C'est à cet évêque de Patara, au $\mathrm{IV}^{\mathrm{e}}$ siècle, que ce texte fut faussement attribué, alors qu'il fut en réalité rédigé à la fin du $\mathrm{VII}^{\mathrm{e}}$ siècle, en Syrie, au moment de l'invasion islamique. Voir Claude Taviani et Huguette Taviani-Carozzi, La Fin des temps, op. cit, p. 101-106. Voir aussi l'article de Camille Rouxpetel, infra, p. 99-120.

${ }^{18} C f$. infra, " oracles païens et prophètes chrétiens », p. 19.

${ }^{19}$ Joachim de Flore (c. 1135-1202), moine cistercien puis abbé du monastère Saint-Jean de Flore, en Calabre, s'est rendu célèbre par ses interprétations eschatologiques des Écritures et fut consulté par les papes et empereurs sur les temps à venir. Refusant le titre de prophète, dont les derniers représentants avaient selon lui disparu avec l'Ancienne Alliance, il se présente non comme celui qui « voit» mais comme celui qui, sachant scruter et méditer les Écritures, reçoit le don de les interpréter : au prophète s'est substitué l'exégète - selon une conception du prophète partagée par nombre de théologiens au Moyen Âge. Joachim de Flore s'est toujours soumis à l'autorité de l'Église, qui reconnut la sainteté de sa vie mais condamna sa théorie sur les trois âges - du Père, du Fils et du Saint-Esprit - exposée dans L'Évangile éternel. 
Flore était invoquée pour justifier les positions prises en faveur de tel ou tel pape ou souverain, dans lequel on voyait le personnage messianique qui, selon la thèse joachimite, devait régénérer l'Église et la chrétienté. À l'inverse, ces mêmes prophéties ont pu alimenter l'opposition à un Frédéric II ou à ses successeurs ${ }^{20}$, présentés successivement comme autant de figures de l'Antéchrist.

L'autre interprétation de l'Apocalypse - celle que l'Église a adoptée - suit la ligne tracée par saint Augustin au livre XX de La Cité de Dieu : le millénarisme y est réfuté comme lecture littérale, «terrestre », au profit d'une lecture dévoilant le sens «spirituel» de la prophétie. L'accent est mis sur l'histoire de l'Église comme «Corps du Christ», «Cité de Dieu» et non comme institution «terrestre» appuyée sur des pouvoirs temporels. La résurrection des élus représente la vie dans le Christ des baptisés, et le millénaire « la plénitude même du temps ${ }^{21}$ » durant lequel se déroule la vie de la Cité de Dieu, jusqu'au jour du Jugement. L'Apocalypse et ses prophéties sont alors moins des prédictions à décrypter pour connaître l'avenir qu'une « révélation », un dévoilement du sens caché de l'histoire, dans laquelle le fidèle doit lire un encouragement à la conversion en vue du retour définitif du Christ. L'histoire apparaît comme le temps où les deux cités sont mêlées, où souffrance et malheurs invitent à reconnaître la culpabilité originelle et à faire pénitence en vue du Salut.

Le $\mathrm{XI}^{\mathrm{e}}$ siècle marque un tournant dans la position des clercs sur ce sujet : la doxa théologique, dans la lignée de saint $\operatorname{Augustin}^{22}$, rejette toute

${ }^{20}$ Cf. Bernhard Töpfer, Das Kommende Reich des Friedens : zur Entwicklung des chiliastischer zukunftshoffnungen im Hochmittelalter, Berlin, Akademie-Verlag, 1964.

${ }^{21}$ Saint Augustin, La Cité de Dieu, dans Euvres complètes, $5^{\text {ème }}$ série, vol. 37 : livres XIX à XXII, Triomphe de la Cité céleste, intr. et notes Gustave Bardy, trad. Gustave Combès, Paris, Desclée de Brouwer, coll. «Bibliothèque augustinienne », 1960, livre XX, chap. 7.

${ }^{22}$ Significatif est à cet égard le Traité sur l'Antéchrist d'Adson de Montier-en-Der, écrit vers 930-992 (De ortu et tempore Antichristi. necnon et tractatus qui ab eo 
interprétation historique de l'Apocalypse. Cette période n'en témoigne pas moins une attention marquée envers les "signes des temps », dans une atmosphère d'attente eschatologique. C'est dans ce contexte que naît la première croisade, à la faveur de laquelle les attentes millénaristes du peuple, qui sont spontanément de nature impériale, sont redirigées par l'Église vers le seul chef et la seule institution capables de conduire les peuples au salut, à savoir le Pape et la Papauté. Le discours clérical interprète donc les phénomènes visionnaires et prophétiques qui entourent cette croisade ${ }^{23}$ dans un sens moral et spirituel : la Jérusalem historique ne sera pas le lieu d'un âge d'or revenu, mais celui du martyre et de la purification en vue d'un Jugement dont on ignore encore la date ${ }^{24}$. Il faut noter cependant que notre connaissance de ces phénomènes et de l'interprétation qu'ils ont reçue est tributaire du point de vue unilatéral des sources : les clercs, écrivant le plus souvent a posteriori, réinterprètent les prophéties dans un sens typologique et spirituel qui n'était pas forcément celui perçu par les laïcs engagés dans la croisade ${ }^{25}$.

dependunt, éd. Daniel Verhelst, Turnholti, Brepols, coll. «Corpus Christianorum Continuatio Medievalis », 45, 1976). Voir Huguette Taviani-Carozzi, La fin des temps, op. cit., p. 34-37.

${ }^{23}$ Sur ce sujet, voir Paul Alphandéry et Alphonse Dupront, La chrétienté et l'idée de croisade [1954-1959], Paris, Albin Michel, 1995.

${ }^{24}$ Le comput effectué par Raoul Glaber sur la date possible de la fin du monde témoigne de la même ambiguïté : on a voulu voir dans ses Histoires, rédigées entre 1030 et 1047, une illustration des «terreurs de l'An mil » (Raoul Glaber, Histoires, trad. Mathieu Arnoux, Turnhout, Brepols, coll. «Miroir du Moyen Âge », 1996). Cependant le débat reste ouvert, dans la mesure où les récits du moine bourguignon témoignent aussi bien d'une attente de la fin des temps que d'un espoir renouvelé devant la réforme monastique et le «blanc manteau d'églises » qui autour de l'An mil a couvert la chrétienté. Voir à ce sujet Huguette Taviani-Carozzi, La fin des temps, op. cit., p. 44-74 et 165-176.

25 Pour une synthèse de ce débat, voir André Vauchez, «Les composantes eschatologiques de l'idée de croisade », Le concile de Clermont de 1095 et la croisade, Actes du colloque universitaire international de Clermont-Ferrand (23-25 juin), Rome, École française de Rome, 1997, p. 233-243 et, sur Guibert de Nogent en particulier, Huguette Taviani-Carozzi, La fin des temps, op. cit., p. 22-29 et p. 113-123. 


\section{Le rôle providentiel du prophète}

Dans la chronique de Geoffroy de Monmouth comme dans les romans en prose $\mathrm{du} \mathrm{XIII}^{\mathrm{e}}$ siècle, Merlin est non seulement l'auteur de prophéties politiques, mais également un personnage providentiel, conseiller des rois choisis par Dieu, chargé de guider ces derniers dans l'accomplissement de leur mission. Ce rôle est particulièrement développé dans le Merlin de Robert de Boron ${ }^{26}$ (rédigé vers 1200-1210): les prophéties à proprement parler y sont réduites, au profit d'un magistral personnage de prophète, dénonciateur du mensonge et de l'injustice en même temps que porte-parole de la Providence; il prophétise les temps bénis qui verront le règne glorieux d'Arthur et l'accomplissement de la mission spirituelle de la Table Ronde. Son action et ses discours sont accompagnés de signes extraordinaires qui cautionnent le caractère surnaturel de sa mission : le prophète est aussi bien celui qui renverse les imposteurs que le protecteur des rois élus par Dieu pour l'exaltation de la chrétienté $^{27}$. En outre il est remarquable que le personnage de Merlin, qui n'est pas un clerc, en vient à faire concurrence, dans son rôle de direction spirituelle du pouvoir temporel, aux ecclésiastiques qui, eux, sont quasiment absents de l'horizon romanesque arthurien. Cependant, ce rôle politique du personnage de Merlin tend à s'estomper au fil des romans, au profit d'un message prophétique tantôt spirituel tantôt profane, mais adressé principalement aux chevaliers individuels pour les guider dans leur

\footnotetext{
${ }^{26}$ Robert de Boron, Merlin, roman du XIII siècle, op. cit.

${ }^{27}$ La suite de ce roman, dite Suite-Vulgate du Merlin ou Les Premiers Faits du roi Arthur, porte les traces d'un certain millénarisme : elle fait du prophète Merlin le protecteur du jeune roi Arthur et le garant de son pouvoir, lequel est appelé à constituer un pilier de la chrétienté. C'est ainsi que la clairvoyance du prophète assure systématiquement à Arthur la victoire contre ses ennemis païens, les envahisseurs saxons, aussi bien que contre les barons révoltés de son royaume, qui refusent l'élection providentielle d'un jeune chevalier d'origine inconnue (Le Livre $d u$ Graal, éd. Daniel Poirion et al., Paris, Gallimard, coll. «Pléiade », t. I, 2001, p. 807-1662).
} 
cheminement. Bien plus, même une fois disparu, le prophète reste présent par sa parole qui résonne dans des manifestations surnaturelles, ou est rappelée par des inscriptions merveilleuses: la prophétie survit en définitive au personnage du prophète.

L'évolution que connaissent le personnage de Merlin et l'utilisation romanesque de ses prophéties rassemble dans le cycle romanesque en $\operatorname{prose}^{28}$ le faisceau des manifestations historiques de la prophétie aux XII ${ }^{\mathrm{e}}$ et $\mathrm{XIII}^{\mathrm{e}}$ siècles. La conception du prophète comme conseiller politique doit beaucoup à l'ancienne confiance de l'Église dans le secours du pouvoir temporel $^{29}$; pour autant elle ne relève pas tout à fait des croyances millénaristes qui étaient projetées sur les empereurs au $\mathrm{XI}^{\mathrm{e}}$ siècle. Le prophète-conseiller politique et personnage providentiel renvoie à une conception de la prophétie qu'ont illustrée plusieurs figures de saints, dont les interventions auprès des grands de ce monde ont porté la voix de Dieu dans les affaires temporelles. Néanmoins, là encore, la figure du prophète médiéval nous est parvenue à travers le prisme du jugement ecclésiastique : bien peu parmi les personnages inspirés qui ont prétendu faire la leçon aux grands ont été reconnus comme prophètes - saint Bernard, sainte Hildegarde de Bingen, sainte Brigitte de Suède, saint François d'Assise au milieu d'une foule de «faux-prophètes » qui, se proclamant inspirés et envoyés par Dieu pour fustiger les crimes des puissants, sont dénoncés par l'Église comme des suppôts de Satan ou simplement comme des fous ${ }^{30}$.

Il faut noter que, pour la doctrine chrétienne, le rôle de conseil dans les affaires temporelles n'est qu'un aspect de la mission du prophète.

\footnotetext{
${ }^{28}$ La rédaction du cycle arthurien en prose s'étend sur tout le XIII ${ }^{\mathrm{e}}$ siècle.

${ }^{29}$ Position qui a été analysée comme «augustinisme politique» (voir sur ce sujet Dominique Boutet, Charlemagne et Arthur, Paris, Champion, coll. " Nouvelle bibliothèque du Moyen Âge », 20, 1992, passim), avec toutes les réserves que l'historiographie contemporaine pose à cette expression.

${ }^{30}$ Sur la figure du faux-prophète, voir dans ce numéro la contribution de Loren Gonzalez, infra, p. 73-98.
} 
Hildegarde de Bingen en représente l'exemple le plus éclairant : elle se dit envoyée par Dieu pour rappeler aux chrétiens et surtout aux détenteurs du pouvoir les exigences de la foi ${ }^{31}$. Visionnaire, elle conçoit le don de prophétie moins comme une capacité de prédiction des événements futurs elle critique violemment les astrologues - que comme une inspiration divine reçue pour comprendre et interpréter les desseins divins cachés, pour proclamer devant les hommes le jugement de Dieu sur leur histoire. Le temps des prophètes n'est selon elle pas terminé ${ }^{32}$; toute parole spirituelle adressée aux hommes est prophétique dans la mesure où elle retrouve la pureté originelle du langage, moyen de communication de l'homme avec Dieu que le péché originel a rendu opaque. C'est dans cette perspective que le prophète est voué à suppléer - notamment dans le contexte de crise qui est celui de l'Église au XII ${ }^{\mathrm{e}}$ siècle - à l'indigence spirituelle des ecclésiastiques incapables de faire face à leurs responsabilités dans les rapports avec les pouvoirs temporels ${ }^{33}$. «Hildegarde se comprend donc elle-même comme une contemplative, i.e. une théologienne, appelée à comprendre et à présenter sous une forme symbolique hautement suggestive les passages fondamentaux de l'histoire du salut ${ }^{34} »$. Ses visions relatives à la fin des temps expriment avant tout l'urgence de cette proclamation, face à la corruption de l'ordre ecclésiastique et dans une

${ }^{31}$ Pour un résumé de cette tradition jusqu'à Hildegarde, voir Lo statuto della profezia nel Medioevo, éd. Gian Luca Potestà et Roberto Rusconi, Bologna, Dehoniane Bologna, coll. « Cristianesimo nella storia », 17, 1996, p. 243-250.

${ }^{32}$ Telle n'est pas la position de Joachim de Flore, qui refuse de se reconnaître prophète et voit sa science exégétique comme une compréhension particulièrement aigüe des Écritures ; cette perspicacité, fruit de la méditation des psaumes et don de Dieu, lui permet de voir ce que les prophètes eux-mêmes n'ont pu comprendre, notamment en ce qui regarde les fins dernières.

${ }^{33}$ Hildegarde réprimande ainsi le pape Anastase IV pour la pusillanimité dont il fait preuve face à l'empereur (André Vauchez, « Le prophétisme médiéval d'Hildegarde de Bingen à Savonarole », Revue de l'Institut catholique de Paris, 68, 1998, p. 169186, en part. p. 117).

${ }^{34}$ Gian Luca Potestà et Roberto Rusconi, Lo statuto della profezia nel Medioevo, op. cit., p. 246 (nous traduisons). 
atmosphère populaire tendue vers une venue prochaine de l'Antéchrist. Hildegarde ne se prononce pas sur cette proximité supposée de la fin des temps, car selon elle la dimension historique de l'attente est secondaire par rapport à sa signification spirituelle ${ }^{35}$. De même, les mouvements prophétiques qui, au XIV ${ }^{\mathrm{e}}$ siècle et lors du Grand Schisme d'Occident (1378-1414), dénonçaient les injustices des pouvoirs en place, attendaient moins la fin des temps qu'un retour à la pureté des origines ${ }^{36}$.

La prédominance dans nos sources du point de vue ecclésiastique amène à nous interroger sur les processus de légitimation de la prophétie, sur les débats entourant son origine et sur la catégorisation dont elle est l'objet.

\section{Origine et légitimité de la prophétie}

\section{Un savoir diabolique?}

Lors li raconte son songe tout ensi coume li rois l'ot songiet. Et quant li rois l'a bien ententu, il se sainne de la merveille qu'il en a, si respont erraument : «Tu n'ies pas hom qu'on doie croire, mais anemis [«diable»], car par sens d'oume [d'homme] ne porroies tu pas savoir si repostes [secrètes] choses que tu m'as chi devisees ${ }^{37} \gg$.

Lorsqu'il profère ses vaticinations, dévoile les pensées des hommes et interprète leurs songes, Merlin est accusé d'être inspiré par le démon. La prophétie est alors remise en cause, non comme prétention indue de l'esprit humain mais comme asservissement d'un homme à Satan, l'adversaire de

\footnotetext{
${ }^{35}$ En cela Hildegarde transmet fidèlement la pensée de saint Augustin, qui jusqu'au siècle suivant et sa relecture par Thomas d'Aquin, domine l'interprétation de l'Apocalypse et de la prophétie chrétienne.

${ }^{36} C f$. André Vauchez, "Jalons pour une histoire du prophétisme chrétien », Rerum gestarum scriptor. Histoire et historiographie au Moyen Âge. Mélanges Michel Sot, dir. Magali Coumert et al., Paris, Presses universitaires de Paris-Sorbonne, 2012, p. 213-224.

${ }^{37}$ La Suite du roman de Merlin, éd. Gilles Roussineau [1996], Genève, Droz, coll. « Textes littéraires français », 972, 2006, § 11.
} 
Dieu qui utilise son savoir pour s'opposer au Créateur. De fait Merlin tient bien sa connaissance des choses passées de son ascendance diabolique : fils du diable qui l'a engendré en une vierge, il est certes racheté par le baptême que lui fait donner sa pieuse mère, mais n'en garde pas moins les capacités reçues de son père, parmi lesquelles la connaissance du passé. La Providence, toutefois, pour s'allier ce personnage dangereux, complète l'héritage diabolique par le don de prophétie, qui consiste en l'occurrence dans la capacité de voir l'avenir ${ }^{38}$. Le roman, en attribuant au savoir de Merlin cette double origine, met en question la légitimité exclusive du prophète inspiré par Dieu ${ }^{39}$.

La fiction romanesque, en cela, rejoint une problématique brûlante dans l'actualité de l'Église des $\mathrm{XII}^{\mathrm{e}}-\mathrm{XIII}{ }^{\mathrm{e}}$ siècles : si la capacité à prophétiser est largement répandue, sa légitimité au regard de l'institution ecclésiastique est le plus souvent suspecte et attribuée à une intervention diabolique. C'est ainsi que le chroniqueur Guillaume de Newburgh ${ }^{40}$, à la fin $\mathrm{du} \mathrm{XII}^{\mathrm{e}}$ siècle, dénonce dans les prophéties de Merlin non pas tant l'invention d'une figure de prophète qui serait sacrilège, mais le détournement par Geoffroy de Monmouth, au service d'une histoire mensongère, de ce fait reconnu par la foi et la raison qu'est la capacité des

38 « [...] vost Nostre Sire que le pechié de sa mere ne li poïst nuire : si li dona pooir et sens de savoir les choses qui estoient a avenir. Par cestes raisons sot cist les choses faites, dites et alees, car il les a et tient de l'enemi ; et le surplus qu'il set des choses qui sont a avenir volt Nostre Sires qu'il seust contre les autres choses qu'il savoit por endroit de la soue partie (Robert de Boron, Merlin, éd. cit., § 10, 1. 24-31.

${ }^{39} \mathrm{La}$ réalisation des desseins divins s'accommode bien du savoir diabolique du prophète : le Merlin, roman providentiel, manifeste un optimisme inébranlable sur la toute-puissance de Dieu, et fait servir même les agissements du diable à la réalisation des desseins de la Providence. D'autres romans (les deux Suites du Merlin, notamment) souligneront au contraire la tension qui persiste à l'intérieur de ce prophète à la double origine : son savoir et ses prophéties ne l'empêcheront pas de se livrer volontairement à l'amour de la femme qui le perdra.

${ }^{40}$ Guillaume de Newburg (1136-1198), chanoine augustin du prieuré Saint-Mary de Newburgh, dans le Yorkshire, écrit son Historia rerum anglicarum vers 1196-1198. 
démons à prévoir et prédire l'avenir ${ }^{41}$. Conformément à la tradition augustinienne $^{42}$, en effet, les médiévaux attribuaient aux démons une capacité de prédire l'avenir, qui n'était pas mise au service de la foi et de l'édification. La prophétie est alors reconnue comme un moyen utilisé par le Diable pour s'opposer à Dieu ${ }^{43}$, et le faux-prophète comme une victime plus que comme un imposteur.

De cette conception de la fausse prophétie comme possession diabolique résultent plusieurs traits communs à l'historiographie, à l'hagiographie et à la littérature. Avant d'être, avec la scolastique, reconnue comme un phénomène intellectuel analysable par le raisonnement philosophie ou théologique ${ }^{44}$, la prescience de l'avenir est considérée comme un désordre de l'esprit qui, d'origine diabolique, ne peut que s'accompagner d'un désordre des mœurs - lubricité, rébellion contre l'autorité, solitude et mode de vie proches de la sauvagerie, etc. La capacité prophétique représente donc, par ses conséquences sur le comportement

${ }^{41}$ «Et hunc quidem Merlinum patre incubo daemone ex femina natum fabulatur, cui propterea tanquam patrissanti excellentissimam atque latissimam tribuit praescientiam futurorum: cum profecto et veris rationibus et sacris litteris doceamur daemones, a luce Dei seclusos, futura nequaquam contemplando praescire: sed quosdam futuros eventus ex signis sibi quam nobis notioribus, conjiciendo magis quam cognoscendo colligere » (Guillaume de Newburg, Historia rerum anglicarum, Rerum anglicarum medii aevi scriptores, or Chronicles and memorials of Great Britain and Ireland during the Middle Ages, éd. Richard Howlett, London, Longman \& Co., Paternoster Row ; Trübner \& Co., Ludgate Hill, Oxford, Parker \& Co., Cambridge, and Macmillan \& Co., Edinburgh, A. \& C. Black et Douglas and Foulis, Dublin, A. Thom \& Co., 1884, vol. I, Proemium p. 12 ; reproduction en fac-similé : Chronicles of the reigns of Stephen, Henry II, and Richard I, Wiesbaden, Krauss Reprint, 1964, vol. I).

${ }^{42} C f$. en particulier saint Augustin, La Cité de Dieu, op. cit., vol. 34 : Impuissance spirituelle du paganisme, 1959, livre IX, chap. 22 et livre X, chap. 32.

${ }^{43}$ La conception de Merlin, par exemple, est le fruit d'un conseil des démons qui, à travers son action sur terre, entendent faire pièce à la Rédemption (Robert de Boron, Merlin, éd. cit., §1). Il est vrai que le personnage ne tient de son ascendance diabolique que la connaissance du passé, non celle de l'avenir qui, elle, lui vient de Dieu. Mais il s'agit bien pour les démons d'accorder à leur engeance une capacité intellectuelle assez exceptionnelle pour que les hommes la créditent d'une origine divine.

${ }^{44}$ Voir à ce sujet la contribution de Valeria Ingegno, infra, p. 29-52. 
social, un danger pour l'ordre établi; d'où la collusion entre pouvoirs ecclésiastiques et séculiers pour lutter contre les fauteurs de troubles. Le lien entre mouvements prophétiques et persécutions civiles ou religieuses a été bien étudié pour les $\mathrm{XIII}^{\mathrm{e}}-\mathrm{XIV}^{\mathrm{e}}$ siècles, dans le contexte des mouvements joachimites ${ }^{45}$.

Il faut noter cependant que le point de vue exclusif des clercs brouille notre perception de ce qu'étaient réellement ces «faux-prophètes »: le même schéma est souvent utilisé pour décrire leur parcours - événement brutal provoquant une rupture avec l'ordre social, refuge dans la vie érémitique, entrée dans la vie publique pour proclamer le message reçu ${ }^{46}$. Bien souvent une simplicité d'esprit est invoquée pour discréditer ces pseudo-vocations; mais la figure du faux-prophète peut alors rejoindre celle du fou ${ }^{47}$ - non pas l'insensé de la Bible mais l'homme humble et méprisé de ses semblables par qui Dieu choisit de se révéler. Les auteurs ecclésiastiques, cependant, ne disposent pas toujours de cet argument

${ }^{45}$ Voir sur ce sujet l'ouvrage classique de Marjorie Reeves, The Influence of Prophecy in the Middle Ages, Oxford, Clarendon Press, 1969; à compléter par Roberto Rusconi, L'Attesa della fine. Crisi della società, profezia ed Apocalisse in Italia al tempo del Grande Scisma d'Occidente (1378-1417), Roma, Istituto Italiano per il Medioevo, 1979.

${ }^{46} \mathrm{La}$ prophétie, d'après son étymologie grecque, est avant tout «proclamation »:

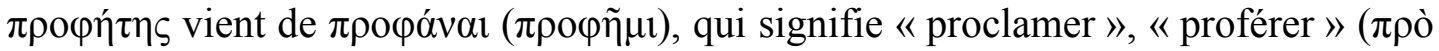
au sens de «pour », « au nom de » et non au sens d'une antériorité chronologique). Certains théologiens et savants - Eusèbe de Césarée, Thomas d'Aquin, Isidore de Séville, Grégoire le Grand - retiennent cependant l'étymon $\pi \rho$ ò- $\varphi \alpha i v \omega$ : on appelle quelqu'un prophète "non quia praedicit uentura, sed quia prodit occulta " (« non parce qu'il prédit les choses à venir, mais parce qu'il met au jour celles qui sont cachées », Grégoire le Grand, Homelia in Ezechielem I, 1, PL 76, col.787, coll. «Corpus Christianorum Series Latina», 142, 5. Nous traduisons). Le sens revient au même, cependant, pour l'argumentation dont il est ici question. $C f$. Claude Carozzi et Huguette Taviani-Carozzi, La fin des temps, op. cit., p. 39. Le personnage de Merlin, tel qu'il est hérité des traditions galloises, répond à ce schéma biographique.

${ }^{47}$ Voir sur ce sujet l'étude lumineuse de Jean-Marie Fritz, Le discours du fou au Moyen Âge. XII ${ }^{e}-X I I I^{e}$ siècles: étude comparée des discours littéraire, juridique et théologique de la folie, Paris, Presses Universitaires de France, coll. «Perspectives littéraires », 1992. Dans les romans arthuriens la voix prophétique de Merlin trouve parfois un relais dans le discours de fous ( $c f$. en particulier La suite du roman de Merlin, éd. cit, § 307). 
ambivalent, de sorte qu'ils sont parfois gênés de devoir reconnaître un haut degré de culture théologique chez ces personnages issus pour une grande part des monastères ou formés dans les écoles cathédrales : leur statut de «faux-prophètes » ne peut provenir d'un dérangement de la raison. Décisive est alors l'accusation portée contre les mœurs de ces déviants, dont la dépravation - réelle ou supposée - devient une preuve incontestable de l'origine diabolique de leurs pouvoirs surnaturels. Le discours véritable autant que l'identité réelle de ces «faux-prophètes » sont donc masqués par le jugement théologique et moral, qui est porté contre leur doctrine et leur personne.

Les discours, néanmoins, se répondent: en les dénonçant comme inspirés par le diable, l'institution ecclésiastique ne fait que renvoyer à ses détracteurs l'accusation que ces derniers lui adressent, celle d'être corrompue par le vice et vendue à Satan; et lorsqu'elle envoie ses accusateurs au bûcher, elle nourrit l'attente eschatologique de ces prophètes et de leurs disciples, lesquels voient dans la persécution une confirmation de leur mission et un signe de la chute prochaine de l'Église instituée. Ce paradoxe a été largement étudié pour des mouvements comme celui des Apostoli $^{48}$, dans l'Italie des XIII ${ }^{\mathrm{e}}-\mathrm{XIV}^{\mathrm{e}}$ siècles.

Alors que l'Église cherche dans l'action du diable la cause de ses troubles internes, les milieux universitaires, autour de théologiens comme Pierre d'Ailly, Henri de Langenstein ou Jean Gerson, mettent au centre de leurs réflexions la probatio spirituum, c'est-à-dire les moyens de démasquer les faux prophètes et de distinguer les révélations divines de

\footnotetext{
${ }^{48}$ Sur ce sujet, voir les travaux de Marjorie Reeves, The Influence of Prophecy in the later Middle Ages, op. cit.; d'Henri de Lubac, La Postérité spirituelle de Joachim de Flore [1979], t. I : « De Joachim à Schelling », Paris/Namur, Lethielleux/Culture et Vérité, coll. «Le Sycomore», 1987; et pour les aspects théologiques de cette question, Gian Luca Potestà et Roberto Rusconi, Lo statuto della profezia nel Medio Evo, op. cit., p. 6.
} 
celles venant du diable ${ }^{49}$.

\section{Divination et pratiques occultes}

La divination, tout comme la prophétie, pose à la foi du chrétien médiéval un problème de légitimité : pour les théologiens ${ }^{50}$, elle se distingue de la prophétie (véritable) par son origine diabolique, dans la mesure où elle force la volonté de Dieu. Il est remarquable que Merlin ne pratique jamais lui-même la divination, alors que des personnages de clercs, à la cour du roi Arthur, y recourent pour tenter de déchiffrer des songes prophétiques ou les vaticinations du prophète. Le roman opère une distinction subtile entre un savoir acquis par l'habileté et l'ingéniosité humaine, et une connaissance surnaturelle reçue d'en-haut: qu'elle soit d'origine divine ou diabolique, la connaissance que Merlin a des choses cachées ne résulte pas d'un apprentissage ni d'une technique, à la différence des « encerchements » des clercs, qui usent de pratiques mises au point par le savoir, la technique et l'expérience ${ }^{51}$.

\section{Oracles païens et prophètes chrétiens}

L'intégration de Merlin à la littérature chrétienne, opérée au $\mathrm{XIII}^{\mathrm{e}}$ siècle par Robert de Boron, qui associe le personnage à une lecture spirituelle des mythes de la Table Ronde et du Graal, témoigne de

${ }^{49}$ Sur ce sujet, voir la contribution de Valeria Ingegno dans ce numéro, infra, p. 29-52.

${ }^{50}$ Richard Trachsler, dans son introduction à un recueil d'études sur la prophétie médiévale, considère la divination comme une catégorie à part entière dans le domaine de la prophétie ( $c f$. Richard Trachsler, «Moult oscure parleüre. Quelques observations sur la prophétie médiévale », Moult obscures paroles, dir. Richard Trachsler, Paris, PUPS, 2009, p. 11-13). Voir, sur ce sujet, la contribution d'Arnaud Sibille dans ce numéro, infra, p. 53-72.

${ }^{51}$ Un exemple frappant en est la consultation que le prince Galehaut des Isles demande à des clercs sur un songe prophétique dont il veut connaître la signification. Maître Hélie de Toulouse, en particulier, rappelle des prophéties de Merlin pour compléter et confirmer ce que lui-même a pu "voir » grâce à ses techniques de divination (Lancelot, éd. Alexandre Micha, Paris/Genève, Droz, coll. «Société des Anciens Textes Français », t. I, chap. IV). 
l'aptitude dont fait preuve le Moyen-Âge à assumer un héritage non chrétien dans une lecture univoque du monde comme révélation de Dieu. Le prophète du Graal, dérivé de ses origines mythologiques - le Myrddin gallois a sans doute plus à voir avec la tradition druidique qu'avec les prophètes de l'Ancien Testament ${ }^{52}$-, suit la même trajectoire que nombre de figures appartenant originellement à des mythologies ou religions païennes, et que le christianisme assume pour les mettre au service de la vérité qu'il proclame. Les oracles païens, notamment ceux de la (ou des) Sibylle(s) ${ }^{53}$, connaissent ainsi une grande fortune auprès des premiers apologistes chrétiens ${ }^{54}$, qui y voient des preuves plus aptes que les prophéties de l'Ancien Testament à susciter la foi chez les païens. Le Moyen Âge a même produit ses propres oracles sibyllins ${ }^{55}$, en les antidatant pour leur donner une autorité prophétique ${ }^{56}$. Parfois les dieux de la

${ }^{52}$ Sur les origines galloises du personnage romanesque de Merlin, voir Le devin maudit. Merlin, Lailoken, Subhuine. Textes et étude, dir. Philippe Walter, Grenoble, Ellug, 1999.

${ }^{53}$ L'Antiquité gréco-romaine connaissait plusieurs Sibylles, selon le témoignage de Varron, que rapportent Lactance et à sa suite saint Augustin (Lactance, Institutions divines, I, 6, éd. Christiane Ingremeau, Paris, Éditions du Cerf, coll. «Sources chrétiennes », 2007 ; saint Augustin, La Cité de Dieu, op. cit., vol. 36 : «Lutte des deux cités », 1960, livre XVIII, chap. 23). La plus connue est la Sibylle tiburtine - de Tibur, ou Tivoli, près de Rome - qui joue un rôle essentiel dans la religion romaine à travers les " livres sibyllins », qu'elle aurait vendus au roi Tarquin le Superbe, et que consultaient en temps de crise des magistrats spéciaux délégués à cet office. Elle est parfois identifiée à la Sibylle d'Érythrée, laquelle serait venue s'installer à Tivoli pour fuir son pays où Apollon lui avait prédit la mort.

${ }^{54}$ Voir notamment Tertullien, Apologétique, XIX, 10, éd. Jean-Pierre Waltzig, Paris, coll. «Les Belles Lettres », 1998 ; saint Justin, Apologie pour les chrétiens, XX et XLIV, éd. Charles Munier, Paris, Éditions du Cerf, coll. « Sources chrétiennes », 2006 ; Lactance, Divinarum Institutionum, IV, IV, XVIII, 15, éd. Pierre Monat, Éditions du Cerf, coll. « Sources chrétiennes », 377, 1992.

${ }^{55}$ En particulier l'Oracle de la Sibylle tiburtine, texte écrit au $\mathrm{XI}^{\mathrm{e}}$ siècle à partir d'un original syrien de la fin du $\mathrm{IV}^{\mathrm{e}}$ siècle ( $c f$. Sibyllinische Texte und Forschungen, op. cit., p. 177-187). Voir à ce sujet l'étude et la traduction de Claude Carozzi et Huguette Taviani-Carozzi, La fin des temps, op. cit., p. 29-33 et p. 125-136.

${ }^{56}$ Hildegarde de Bingen reçut de ses contemporains le titre de "Sibylle rhénane» (cf. Sylvain Gouguenheim, La Sibylle du Rhin. Hildegarde de Bingen, abbesse et prophétesse rhénane, Paris, PUPS, 1996), et Jean Gerson désigna Jeanne d'Arc comme une "Sibylle française" ( $c f$. André Vauchez, "Jeanne d'Arc et le 
mythologie gréco-romaine rejoignent seulement de manière indirecte la culture chrétienne, comme dans ces mythes de fondation où les historiographes invoquent l'autorité d'oracles païens aux origines de la conquête de terres devenues par la suite chrétiennes. Il en va notamment ainsi des mythes relatifs à la fondation par Brutus de ce qui deviendra la Grande-Bretagne, dont l'origine est attribuée à un oracle de Diane ${ }^{57}$.

Le rôle que jouent les prophéties païennes dans l'ensemble de la révélation chrétienne a été justifié d'un point de vue théologique par saint Augustin, à l'aide d'arguments dont la fortune médiévale n'a pas encore été entièrement explorée mais qui peut s'avérer féconde pour l'étude de la littérature ou des mythologies médiévales. Il s'agit d'un côté de sa conception de la Providence, qui sera reprise par tout le Moyen Âge: l'économie du Salut est ordonnée de manière à ce que la venue du Christ soit annoncée principalement au peuple juif, mais aussi parmi les païens, ce afin de les préparer à reconnaître le Messie au moment de sa venue ${ }^{58}$. Augustin également développe un exemple vétéro-testamentaire voué lui aussi à une grande fortune - mais mieux étudié ${ }^{59}-$, dans l'expression consacrée «les dépouilles des Égyptiens »: il s'agit pour lui d'illustrer l'usage que le chrétien lecteur des Écritures peut faire de la culture païenne, à partir de l'argument selon lequel toute vérité, même exprimée par ceux qui méconnaissent le vrai Dieu, appartient de droit au chrétien ${ }^{60}$. La foi

prophétisme féminin des $\mathrm{XIV}^{\mathrm{e}}$ et $\mathrm{XV}^{\mathrm{e}}$ siècles », dans Les Laïcs aux Moyen Âge. Pratiques et expériences religieuses, Paris, Éditions du Cerf, 1987, p. 277-286; Colette Beaune, Jeanne d'Arc, Paris, Perrin, 2004).

${ }^{57}$ Voir à ce sujet Émilie Deschellette, «L'identité à l'épreuve du mythe : la fabrique des origines, d'Énéas à Brutus », Questes, n 24, 2012, "L'identité », p. 66-95.

${ }^{58}$ Augustin tient ce raisonnement à propos des prophéties de la Sibylle d'Érythrée ( $\mathrm{La}$ Cité de Dieu, op. cit., XVIII, 23), et de la IV églogue de Virgile (ibid., vol. 34, X, 27). Il reproche au philosophe Porphyre, en particulier, de ne pas avoir reconnu le Christ dans la prophétie de la Sibylle rapportée par Virgile.

${ }^{59}$ Cf. Michel Zink, Poésie et conversion, Paris, Presses Universitaires de France, 2003, p. 19-27.

${ }^{60}$ Sicut enim Aegyptii [...] vasa atque ornamenta de auro et de argento et vestem, quae 
chrétienne, telle qu'elle est formulée par le Père de l'Église qui modela en grande partie la pensée médiévale, n'interdit donc pas la fréquentation des doctrines, religions et mythologies païennes, à condition que le chrétien soit assez éclairé pour n'en retenir que le vrai.

\section{L'écriture prophétique}

\section{Merlin et Blaise : le prophète et sa plume vivante}

\section{Le Livre des prophéties de Merlin que tout le Moyen Âge lit,} interprète et réécrit ${ }^{61}$ a son histoire elle-même écrite à l'intérieur des romans arthuriens. Le processus et les circonstances de sa rédaction sont

ille populus exiens de Aegypto sibi potius tamquam ad usum meliorem clanculo vindicavit, non auctoritate propria, sed praecepto Dei, ipsis Aegyptiis nescienter commodantibus ea quibus non bene utebantur; sic doctrinae omnes Gentilium non solum simulata et superstitiosa figmenta gravesque sarcinas supervacanei laboris habent, quae unusquisque nostrum, duce Christo, de societate Gentilium exiens, debet abominari atque devitare, sed etiam liberales disciplinas usui veritatis aptiores et quaedam morum praecepta utilissima continent, deque ipso uno Deo colendo nonnulla vera inveniuntur apud eos. Quod eorum tamquam aurum et argentum quod non ipsi instituerunt, sed de quibusdam quasi metallis divinae providentiae, quae ubique infusa est, eruerunt («Voyez les Égyptiens : [...] ils avaient des vases et des bijoux d'or et d'argent ainsi que des vêtements : or ce peuple, à sa sortie d'Égypte, s'appropria en cachette ces richesses, dans l'intention d'en faire un meilleur usage, et cela non pas de sa propre autorité, mais sur l'ordre de Dieu, les Égyptiens euxmêmes leur ayant confié par inadvertance ces biens dont ils faisaient un mauvais usage. Il en va de même de tous les enseignements des païens : ils comportent sans doute des fictions où règnent le mensonge et la superstition et un lourd bagage de travaux superflus que chacun de nous, quand, sous la conduite du Christ, il sort de la société des païens, doit avoir en abomination et éviter ; mais ils comportent aussi les arts libéraux mieux appropriés à la pratique de la vérité, et des préceptes moraux très utiles; et touchant le culte du Dieu unique, on trouve chez eux quelques vérités. C'est là comme leur or et leur argent, qu'ils n'ont pas créés eux-mêmes, mais qu'ils ont extraits comme de certains gisements fournis par la divine Providence qui est partout répandue »). Saint Augustin, De Doctrina christiana, dans Euvres de saint Augustin, $1^{\text {ère }}$ série, vol. 11/2, éd. Jacques Martin, intr. et trad. Madeleine Moreau, notes Isabelle Bochet et Goulven Madec, Paris, Institut d'études augustiniennes, coll. «Bibliothèque augustinienne », 1997, livre II, chap. 40, § 60. Voir également ibid., II, 18, 28.

${ }^{61}$ Voir en particulier la compilation, à la fin du XIII ${ }^{\mathrm{e}}$ siècle, du franciscain italien Richard d'Irlande (Nathalie Koble, Les " Prophesies de Merlin » en prose. Le roman arthurien en éclats, Paris, Champion, coll. «Nouvelle Bibliothèque du Moyen Âge », 92, 2009). 
mis en scène au milieu du récit des aventures chevaleresques et courtoises. À la cour d'Arthur, d'abord, un livre est sur les ordres du roi exclusivement dédié à la consignation des prophéties de Merlin. Celles-ci, en effet, qu'elles soient proférées par Merlin lui-même ou rapportées par un personnage qui en a eu connaissance, ou encore manifestées de manière surnaturelle - voix désincarnée, inscription merveilleuse - ne sont généralement pas comprises sur le moment, de sorte que le roi les fait transcrire fidèlement afin qu'au temps venu de leur réalisation tous puissent reconnaître la signification et la véracité des dires du prophète.

Dans un espace narratif distinct, c'est Merlin lui-même qui fait consigner par écrit ses propres prophéties. À l'écart de la cour, de ses aventures guerrières, chevaleresques ou courtoises, le prophète retrouve au fond de la forêt de Northumberlande son maître Blaise ${ }^{62}$ à qui il dicte, avec les événements dont il a été témoin, l'interprétation prophétique que luimême en donne - ou encore profère de nouvelles prophéties dont Blaise, pas plus que les personnages de la cour d'Arthur, ne comprend la signification ni la portée. Ce geste de faire mettre par écrit une révélation d'origine divine ${ }^{63}$ rappelle l'attitude du prophète Jérémie, à qui le Seigneur demande d'écrire un livre des prophéties qu'il lui a données :

Factum est verbum hoc ad Hieremiam a Domino dicens tolle volumen libri et scribes in eo omnia verba quae locutus sum tibi [...] vocavit ergo Hieremias Baruch filium Neriae et scripsit Baruch quos locutus est ad eum in volumine libri (Vulgate, Hieremias propheta XXXVI, 1-2 et 4) ${ }^{64}$.

${ }^{62}$ Ce Blaise est désigné dans le Merlin comme un "preudome », c'est-à-dire en l'occurrence à la fois un prêtre et un saint homme, qui est le confesseur de la mère de Merlin. Dans les autres romans il est plutôt représenté comme un ermite.

${ }^{63}$ Merlin réaffirme à plusieurs reprises l'origine divine de son savoir, et la mission providentielle qui lui incombe en conséquence ( $c f$. par exemple Les premiers faits $d u$ roi Arthur, dans Le Livre du Graal, t. I, éd. Daniel Poirion et al., Paris, Gallimard, coll. « Pléiade », 2001, § 250).

${ }^{64}$ « Le Seigneur dit à Jérémie : "Prends un rouleau et écris toutes les paroles que je t'ai adressées [...]". Jérémie appela Baruch, fils de Nériyya, qui sous sa dictée écrivit sur 
Ce geste donne à l'acte d'écrire - ou de dicter - l'auctoritas propre aux Écritures. En témoigne également, dans L'Estoire del Saint Graal ${ }^{65}$, la mise en scène qui tient lieu de prologue : le narrateur s'y montre recevant du Christ lui-même un message à transmettre, et qui correspond au roman qu'il entreprend de raconter ${ }^{66}$. À imiter ainsi, au nom de l'auteur ou d'un personnage jouant le rôle d'un auteur, le geste des auteurs sacrés de la Révélation chrétienne, le roman se situe - bien avant le poète vates des romantiques - dans la lignée des prophètes inspirés par Dieu pour conter aux hommes l'histoire du salut, passée, présente ou encore à venir ${ }^{67}$. Les recherches autour de la figure d'un Merlin auteur sont nombreuses ${ }^{68}$, mais cette figure d'un prophète perpétuant par le roman la tradition chrétienne d'évangélisation - ou du moins d'édification - mérite d'être approfondie ${ }^{69}$.

\section{Prophétie et obscurité}

un rouleau toutes les paroles que le Seigneur avait adressées au prophète » (Jérémie 36, 2 et 4. Traduction de la Bible de Jérusalem).

${ }^{65} \mathrm{Ce}$ roman, bien qu'écrit après le Merlin, précède celui-ci dans l'ordre chronologique du cycle.

${ }^{66}$ Cf. L'Estoire del Saint Graal, éd. Jean-Paul Ponceau, Paris, Champion, 1997, § 1-5.

${ }^{67}$ Voir l'éclairante formulation que Nathalie Koble donne de cette interprétation, à propos des Prophecies de Merlin de Richard d'Irlande : « [...] la transmission de la parole révélée fait l'objet d'une construction fictionnelle particulièrement élaborée. Le prosateur a choisi d'introduire son univers prophétique et didactique en l'intégrant dans une logique proprement romanesque : la parole de Merlin sera recueillie dans un livre, dont les aventures seront déterminées par la destinée du prophète dans l'univers arthurien. Jour après jour, chapitre après chapitre, le récit détaille les événements qui accompagnent cette mise en écrit du discours sage, traité comme une aventure romanesque parmi d'autres. Aucune prophétie n'est ainsi isolée du cadre qui définit son écriture et sa réception : le lecteur peut toujours reconstituer, pour chacune d'entre elles, le temps et le lieu de son énonciation et les conditions de sa transcription. ॥ (Nathalie Koble, Les Prophecies de Merlin. Le roman arthurien en éclats, Champion, 2009, p. 47).

${ }^{68}$ Voir en particulier Kate Cooper, « Merlin romancier : paternity, prophecy and poetics in the Huth Merlin », Romanic Review, t. 77, 1986, p. 1-24 ; Larry S. Crist, "Les livres de Merlin », Mélanges Pierre Jonin, Senefiance, 7, 1979, p. 197-210 [reproduit dans Fils sans père, dir. Denis Hüe, Orléans, Paradigme, 2000, p. 77-87].

${ }^{69}$ Paul Zumthor a posé les jalons d'une telle enquête, dans son ouvrage ancien mais d'une grande hauteur de vue (Paul Zumthor, Merlin le Prophète, un thème de la littérature polémique, de l'historiographie et des romans, Genève, Payot, 1943 ; réimpr. Genève, Slatkine, 1973). 
La prophétie de Merlin, comme celle de l'Ancien Testament, comme le langage des rêves ${ }^{70}$, est imagée, obscure, incompréhensible. Si l'usage des références animales trouve un sens dans le contexte de l'engouement médiéval pour les bestiaires, c'est du côté de la réflexion théologique sur la prophétie scripturaire qu'il faut chercher une théorisation médiévale du langage obscur. Henri de Lubac a montré de manière éclairante le fonctionnement et l'importance de la théorie des quatre sens de l'Écriture ${ }^{71}$; celle-ci peut être appliquée au roman, où tantôt Merlin, tantôt des ermites se présentent opportunément à chaque fois qu'un personnage échoue à interpréter lui-même un songe apparemment prophétique, ou un avertissement reçu à travers des manifestations surnaturelles. Les prophéties de Merlin rapportées par Geoffroy de Monmouth ont suscité bien des vocations d'exégètes au long du Moyen Âge - à l'exception notable du premier traducteur de Geoffroy, Wace, qui dénonce la vanité de ceux qui prétendent comprendre la signification des vaticinations merliniennes, et s'abstient pour sa part de toute interprétation ${ }^{72}$. Toutefois, il est une voie qu'il reste à exploiter : l'interprétation que saint Augustin donne de l'obscurité des Écritures, et en particulier de ses prophéties, apporte à ce sujet des lumières dont l'héritage médiéval n'a pas fini d'être étudié. Dans le De Doctrina christiana, Augustin montre que l'obscurité des prophéties vétéro-testamentaires est voulue par Dieu dans un but à la

${ }^{70}$ Sur l'interprétation des rêves au Moyen Âge, voir Simone Collin, « L'emploi des clés des songes dans la littérature médiévale », Bulletin philologique et historique, année 1967, 2, 1969, p. 851-866 ; Steven R. Fischer, « Dreambooks anf the interpretation of medieval literary dreams ", Archiv fûr Kulturgeschichte, 65, 1983, p. 1-20; Steven F. Kruger, Dreaming in the Middle Ages, Cambridge, Cambridge University Press, 1992.

${ }^{71}$ Cf. Henri de Lubac, Exégèse médiévale. Les quatre sens de l'Écriture, Paris, Aubier, coll. « Théologie », 1959.

72 «Dunc dist Merlin les prophecies /Que vus avez, ço crei, oïes, /Des reis ki a venir esteient $/ \mathrm{Ki}$ la terre tenir deveient. $/ \mathrm{Ne}$ vuil sun livre translater/Quant jo nel sai interpreter. /Nule rien dire ne vuldreie /Que si ne fust cum jo dirreie » (Wace, Le Roman de Brut, éd. Ivor D. O. Arnold, Paris, coll. "Société des Anciens textes Français », 1938-1940, 2 vol., t. II, v. 7535-7542). 
fois moral et pédagogique. D'un côté leur caractère incompréhensible traduit l'ineffabilité de Dieu et abaisse l'orgueil de l'exégète qui prétendrait tout comprendre ${ }^{73}$. D'un autre côté le lecteur, stimulé par la difficulté, prendra plaisir à la vaincre, et cet effort le détournera de l'ennui, ennemi de la connaissance de Dieu :

Sed multis et multiplicibus obscuritatibus et ambiguitatibus decipiuntur qui temere legunt, aliud pro alio sentientes; quibusdam autem locis quid vel falso suspicentur non inveniunt, ita obscure dicta quaedam densissimam caliginem obducunt. Quod totum provisum esse divinitus non dubito, ad edomandam labore superbiam et intellectum a fastidio renovandum, cui facile investigata plerumque vilescunt. [...] Nunc tamen nemo ambigit et per similitudines libentius quaeque cognosci et cum aliqua difficultate quaesita multo gratius inveniri. Qui enim prorsus non inveniunt quod quaerunt, fame laborant ; qui autem non quaerunt, quia in promptu habent, fastidio saepe marcescunt: in utroque autem languor cavendus est. Magnifice igitur et salubriter Spiritus Sanctus ita Scripturas sanctas modificavit, ut locis apertioribus fami occurreret, obscurioribus autem fastidia detergeret. Nihil enim fere de illis obscuritatibus eruitur, quod non planissime dictum alibi repperiatur ${ }^{74}$.

${ }^{73}$ Hildegarde de Bingen reprend pour partie cette interprétation, lorsqu'elle fait de l'obscurité des Écritures et des difficultés de l'homme à comprendre le langage de Dieu une conséquence de la chute, qui rendit l'homme inapte au langage spirituel. La prophétie est alors la parole qui, purifiée, retrouve la fonction originelle du langage (André Vauchez, Saints, prophètes, visionnaires, op. cit.).

${ }^{74}$ « Mais de telles obscurités et ambiguïtés diverses abusent ceux qui la lisent à la légère, prenant un sens pour un autre. En certains passages ils ne voient pas quelle conjecture, même fausse, ils pourraient faire, et de cette façon, sur des textes obscurs ils répandent les plus épaisses ténèbres. Tout cela, je n'en doute pas, a été disposé par le plan divin pour dompter l'orgueil par l'effort, et sauver de l'ennui l'intelligence de celui pour qui les recherches faciles n'ont généralement plus de prix. [...] Et pourtant, nul ne conteste qu'on apprend plus volontiers toute chose à l'aide de comparaisons, et que ce qu'on a cherché avec quelque difficulté se découvre avec beaucoup plus de plaisir. Qui, en effet, ne trouve pas d'emblée ce qu'il cherche souffre de la faim ; mais qui ne le cherche pas, parce qu'il l'a sous la main, dépérit souvent de dégoût. Dans les deux cas, c'est la mollesse qu'il faut éviter. Aussi 
L'obscurité du langage prophétique trouve donc sens dans une perspective édifiante : le lecteur s'attachera d'autant plus fermement à la vérité qu'il aura mis plus longtemps à la découvrir. Mais elle trouve également sens d'un point de vue qui est a priori étranger au rapport des médiévaux - en particulier des clercs - à leur texte fondateur ${ }^{75}$, et qui pourtant est exprimé dans ce texte par Augustin et permettrait sans doute d'expliquer en partie la fascination des intellectuels du Moyen Âge pour le langage crypté : il s'agit du plaisir proprement littéraire, part mystérieuse et certes non négligeable pour Augustin de la joie procurée par la Parole de Dieu :

Quid est ergo, quod, si haec quisquam dicat ${ }^{76}$, minus delectat audientem quam si ad eundem sensum locum illum exponat de Canticis canticorum [...]? Et tamen nescio quomodo suauius intueor sanctos, quando quasi dentes uideo [...]. Sed quare suavius videam, quam si nulla de divinis Libris talis similitudo promeretur, cum res eadem sit eademque cognitio, difficile est dicere et alia quaestio est ${ }^{77}$.

l'Esprit Saint a-t-il disposé les saintes Écritures d'une façon si magnifique et si salutaire que dans les passages plus clairs il va au devant de la faim, et dans les plus obscurs il élimine le dégoût. De fait, on ne relève à peu près rien dans ses obscurités qui ne se trouve exprimé très simplement ailleurs » (De Doctrina christiana livre II, chap. $6, \S 7$ et 8).

${ }^{75}$ La thèse en cours d'Hélène Dupraz-Rochas (Le plaisir littéraire dans la littérature narrative en vers autour de 1200, sous la direction de Dominique Boutet, Université de Paris IV-Sorbonne) devrait sur ce sujet apporter à la recherche une matière et des clés d'interprétation qui lui manquent actuellement.

${ }^{76}$ Augustin vient d'exposer de manière transparente un aspect de la doctrine du baptême.

${ }^{77}$ «Comment se fait-il que si l'on parle ainsi, on plaît moins à l'auditeur que si, pour exprimer la même idée, on lui exposait le passage du Cantique des Cantiques [...]? Et pourtant, je ne sais pourquoi, je contemple les saints avec plus d'agrément quand je les vois, comme s'ils étaient les "dents" de l'Église [...]. Mais pourquoi cette vision $m$ 'est-elle plus agréable que si nulle comparaison de ce genre, tirée des Livres saints, ne m'était présentée, alors qu'il s'agit du même fait et de la même notion ? Il est difficile de le dire, et c'est une autre question » (De Doctrina christiana, op. cit., livre II, chap. VI, § 8). Cet aspect du plaisir esthétique est présent dans le texte précédemment cité avec les adverbes et locutions adverbiales libentius («plus volontiers »), multo gratius (« avec beaucoup plus de plaisir»), magnifice (« d'une 
L'étude de la prophétie médiévale doit donc s'arrêter à la question du langage, de ses ambiguïtés, de son attrait, du rôle qui lui est attribué dans le salut chrétien : les médiévaux l'analysent dans la perspective augustinienne d'une nature déchue, qui a perdu ses capacités originelles mais est appelée à les retrouver sous la conduite de la parole divine.

façon magnifique ») et, par contraste sémantique, les syntagmes a fastidio renovandum ("sauver de l'ennui »), fastidia detergeret (« qu'il élimine le dégoût »). Voir aussi La Cité de Dieu, op. cit., XVIII, 28 (à propos d'une parole prophétique d'Osée): Hoc si adhuc velimus exponere, eloquii prophetici obtundetur sapor («Vouloir expliquer davantage ces paroles serait émousser la saveur du langage prophétique »). La réception médiévale de la pensée augustinienne est à cet égard encore à approfondir. 